\title{
USING CATEGORICAL DEA TO ASSESS THE EFFECT OF SUBSIDY POLICIES AND TECHNOLOGICAL LEARNING ON R\&D EFFICIENCY OF IT INDUSTRY
}

\author{
Li-Ting $\mathrm{YEH}^{1}$, Dong-Shang $\mathrm{CHANG}^{2 *}$ \\ ${ }^{1}$ Department of Cooperative Economics and Social Entrepreneurship, Feng Chia University, \\ No. 100 Wenhwa Rd, Seatwen Taichung, 40724, Taiwan, R.O.C \\ ${ }^{2}$ Department of Business Administration, National Central University, No. 300, Zhongda Rd., \\ Zhongli District, Taoyuan City 32001, Taiwan, R.O.C
}

Received 15 January 2019; accepted 22 September 2019

\begin{abstract}
Government subsidies are an important policy tool that can help firms develop technological learning, and this technological learning effect plays a key role in firms' research and development $(\mathrm{R} \& \mathrm{D})$ efficiency. Thus, this study develops a two-stage approach to illustrate the effect of subsidy policies and technological learning on R\&D efficiency in the information technology (IT) industry. The technological learning effect in 128 firms in the IT industry from 2008 to 2015 was measured using the learning experience curve. Subsequently, government R\&D subsidy intensity was considered as a categorical variable, and this estimated result was treated as an intangible input into a data envelopment analysis (DEA) structure to evaluate R\&D efficiency in 2015. This study makes three major contributions. First, the developed approach incorporates the effect of subsidy policies and technological learning into the DEA structure. Second, the empirical results demonstrate the appropriateness of incorporating subsidy policies and technological learning into evaluations of R\&D efficiency. Finally, our results identify the key sources of inefficiency as a shortfall in the number of patents and a lack of technological learning. Based on these key findings, some improved strategies were recommended to decision makers.
\end{abstract}

Keywords: data envelopment analysis, government subsidies, information technology industry, learning experience curve, technological learning, $R \& D$ efficiency.

JEL Classification: C32, C67, H20, L52, L63.

\section{Introduction}

Research and development (R\&D) has been one of the key driving forces of technological progress, and it contributes to increased productivity and profit growth (Barreto \& Kypreos, 2004). The main source of productivity growth in the information technology (IT) industry is the technological learning effect (Patibandla \& Petersen, 2002). Technological learning

${ }^{\star}$ Corresponding author. E-mail: changds@mgt.ncu.edu.tw

This is an Open Access article distributed under the terms of the Creative Commons Attribution License (http://creativecommons. org/licenses/by/4.0/), which permits unrestricted use, distribution, and reproduction in any medium, provided the original author and source are credited. 
has been defined as the learning experience by which a technology-oriented firm can create, renew, and upgrade its intangible capital based on its stock of knowledge-based resources (Hitt, Ireland, \& Lee, 2000). Thus, the technological learning effect can represent the cumulative stock of knowledge, which is a type of intangible capital (Coccia, 2009). Dodgson (1991) considered technological learning as encompassing a firm's build and supplement the knowledge base that is related to their technologies, products, services and processes and how they develop and use the broad skills of their workforce. The learning experience may also involve a new technology or be a product of an experience that allows employees to obtain the required knowledge (Ngwenyama, Guergachi, \& McLaren, 2007). This knowledge can be accumulated through continuous learning over a certain period of time, and this cumulative result can be considered a stock of knowledge at a certain time point. Previous studies have shown that technological learning can reduce $\mathrm{R} \& \mathrm{D}$ expenditures and further improve R\&D efficiency over time (Wang, H. Li, R. Li, B. Li, \& Zhu, 2016). A firm's technological learning experience curve is determined both by knowledge accumulation resulting from informal $\mathrm{R} \& \mathrm{D}$ (through gaining experience from an increase in production) and by formal $\mathrm{R} \& \mathrm{D}$ expenditures (which indicate the level of past support). Technological learning can be investigated using a learning experience curve, which is one of the tools used to quantify the technology developed through the technology learning process (Nakata, Sato, Wang, Kusunoki, \& Furubayashi, 2011).

To gain sustainable competitive advantage, many governments are interested in attracting new technology investment projects by offering various incentives (Chen, Wen, Wang, \& Nie, 2017; Czarnitzki \& Hussinger, 2018; Okamuro \& Nishimura, 2018; Yang, Nie, Liu, \& Shen, 2018). With these incentives, governments have invested heavily in R\&D activities in the IT industry (Sohn \& Moon, 2004). Government subsidy policies are not controlled by corporate decision makers. Because the $\mathrm{R} \& \mathrm{D}$ subsidies that are sponsored by the government are only a small portion of R\&D expenditures at most firms, not all output comes from government financial support (Hsu \& Hsueh, 2009). However, government financial support for R\&D can facilitate firms' technological learning, which enhances firms' ability to develop R\&D output (patent counts) and build their core technological competencies (Lall, 2000). Government subsidy policies may have benefits for local firms' R\&D efficiency through their positive effect on the firms' technological learning capabilities.

Assessing R\&D efficiency helps inefficient firms identify the best practices for benchmarking and provides these firms with appropriate strategies to improve their efficiency by emphasizing areas of weakness. Data Envelopment Analysis (DEA) structure is a nonparametric technique which has advantages over other mathematical methods in assessing the efficiency of R\&D activities. The nonparametric approach does not need to impose explicit production functions or an explicit distributional assumption. In addition, DEA can simultaneously handle multiple inputs and multiple outputs that are expressed in different units of measurement, and it allows each decision-making unit (DMU) to choose its most beneficial weights for the inputs and outputs under its own circumstances. The estimation of relative R\&D efficiency using the DEA model should be made in a homogeneous subsidy policy because the lack of categorical environmental variables could lead to results of the efficiency scores that may reflect the underlying differences in the business operating environment rath- 
er than their actual inefficiency (Haas \& Murphy, 2003). The categorical DEA model is considered in this paper such that each category of DMU is evaluated against DMUs in the same environmental category or in categories that face the worst business operating environment.

In summary, government financial subsidies can help give firms more technological learning opportunities, and this technological learning effect has a significant impact on firms' $R \& D$ efficiency. Currently, there is a gap in the literature concerning how to integrate the effect of subsidy policies and technological learning into R\&D efficiency measures. Thus, existing methodologies are insufficient for investigating this relationship and discussing the methods of formulating improved R\&D strategies for inefficient firms. The contribution of this study is threefold. First, this study develops a methodology that integrates the effect of subsidy policies and technological learning into the categorical DEA model. Second, this study describes the empirical application of the proposed DEA model to evaluate R\&D efficiency in IT industry. Finally, the results obtained from the DEA approach can help inefficient firms to identify the key sources of inefficiency and provide appropriate mechanisms to enhance their R\&D efficiency.

\section{Methodology}

The main contribution of this study is to develop a methodology that integrates the effect of technological learning $\left(\widehat{x^{T L}}\right)$ and subsidy policies $\left(z_{h}\right)$ into the categorical DEA model. Through continuous learning by doing, firms possess the ability to effectively initiate the accumulation and creation of intellectual capital or knowledge energy (Peters \& Waterman, 1985). Learning $\left(\widehat{x^{T L}}\right)$ in the form of acquiring technological knowledge is valuable and unique because it leads to product innovation (Kessler, Bierly, \& Gopalakrishnan, 2000). Technological innovation is considered to be an important driving force in economic growth $\left(y_{1}\right)$ (Nie \& Wang, 2019; Nie, Wang, Chen, \& Yang, 2018; Wang, Nie, Peng, \& Li, 2017). More learning input $\left(\widehat{x^{T L}}\right)$ can improve technology, increase future economic outputs $\left(y_{1}\right)$ and result in superior efficiency (Lyu, Bian, \& Yu, 2018). The technological learning effect $\left(x^{T L}\right)$ was thus treated as an intangible input in the proposed DEA methodology. Generally, DEA models are assumed to be based on a set of observed variables. The technological learning effect $\left(\widehat{x^{T L}}\right)$ is an unobservable variable and reflects the cumulative knowledge that results over a particular period of time. This paper adopts a two-stage approach. In the first stage, the technological learning effect $\left(x^{T L}\right)$ at time $t$ is estimated for each firm, i.e., DMU, respectively. The prior literature such as Chung (2001), Lyu et al. (2018), Ngwenyama et al. (2007), Wong \& Govindaraju (2012) reveals that the ordinary least-squares method is widely used to estimate learning effects. Especially, Papineau (2006) showed that the panel data regression approach may suffer from correlation between the firm specific effects captured in the error term and other variables. Therefore, following the existing studies, this study uses the ordinary least-squares method to estimate the technological learning effect $\left.\widehat{\left(x^{T L}\right.}\right)$ rather than the panel data regression approach. In the second stage, the estimated technological learning effect $\left(x^{T L}\right)$ is treated as an intangible input into the DEA model evaluations.

Environmental categorical variables have a direct influence on efficiency such that if these variables are ignored, the efficiency measurements will be biased. These results will not pro- 
vide appropriate strategies for inefficient DMUs. For subsidy policies that are not under the direct control of corporate decision makers, the categorical DEA model may be an appropriate approach for $R \& D$ efficiency evaluation because through this approach, one can fairly evaluate each inefficient firm included in the study. This model is based on multiple DEA model evaluations and expands the reference set of DMUs in increments of one environmental category. Government R\&D subsidy intensity $\left(z_{h}\right)$ is thus considered an environmental categorical variable in the proposed DEA methodology.

\subsection{Estimated technological learning effect}

Technological learning phenomena were first observed by Wright (1936), who found that unit costs declined significantly when accumulated knowledge stock was measured based on the cumulative volume of production or technology (the output). Wright's one-factor learning experience curve, which considered cumulative output, is among the best empirically corroborated models that characterize technological change in industry (Papineau, 2006). Several authors, however, have argued that Wright's one-factor curve may not be suitable for analyzing technologies. For instance, Söderholm and Sundqvist (2007) identified the problem of omitted variable bias: R\&D cost reductions can also result from past efforts, leading to an overestimation of technological learning if $R \& D$ expenditures (which indicate the level of effort) are ignored. To solve this problem, Kouvaritakis, Soria, \& Isoard, (2000) introduced a two-factor curve to explicitly examine $R \& D$ efforts in technological learning. The twofactor learning experience curve adds cumulative $R \& D$ expenditures as a control variable and processes the omitted variable bias. This two-factor learning experience curve has been commonly used to empirically assess technological learning and is generally recognized as the basic model to measure such learning.

The two factors are the cumulative effect of technological learning $\left(\widehat{x^{T L}}\right)$ and cumulative $\mathrm{R} \& \mathrm{D}$ expenditures $\left(C E_{t}\right)$. R\&D expenditures $\left(x_{1}\right)$ are invested in $\mathrm{R} \& \mathrm{D}$ activities, which are intended to produce valuable knowledge. Because this knowledge is unobservable, the number

of patents $\left(y_{1}\right)$, which is a quantitative indicator, is considered an acceptable estimate of R\&D output (Wang \& Huang, 2007). Cumulative patents $\left(\sum_{s=0}^{s=t} y_{1 s}\right)$ represent knowledge from which future patentable ideas can be effectively derived. As R\&D expenditures $\left(x_{1}\right)$ are a main driver of patenting $\left(y_{1}\right)$, the cumulative effect of technological learning $\left(x^{T L}\right)$ is purged from the impact of R\&D expenditures $\left(x_{1}\right)$. Accordingly, $\mathrm{R} \& \mathrm{D}$ expenditure $\left(x_{1}\right)$ may enhance a firm's technological learning effect $\left(\widehat{x^{T L}}\right)$ and increase the potential for these effects to be converted into patents $\left(y_{1}\right)$. This means that more $\mathrm{R} \& \mathrm{D}$ expenditures $\left(x_{1}\right)$ may lead to more patents $\left(y_{1}\right)$ or that $\mathrm{R} \& \mathrm{D}$ expenditures $\left(x_{1}\right)$ are an additional factor that increases the probability that firm knowledge not related to $\mathrm{R} \& \mathrm{D}$ is turned into patents $\left(y_{1}\right)$. Thus, the cumulative past $\mathrm{R} \& \mathrm{D}$ expenditure $\left(C E_{t}\right)$ is considered a control variable. The two-factor learning experience curve is thus specified as

$$
\frac{1}{x_{1 t}}=A \times\left(\sum_{s=1}^{s=t} y_{1 s}\right)^{a} \times\left(C E_{t}\right)^{b}
$$


where $x_{1 t}$ equals the $\mathrm{R} \& \mathrm{D}$ expenditures in period $t, A$ denotes a constant parameter, $y_{1}$ depicts the number of patents, $\sum_{s=1}^{s=t} y_{1 s}$ measures the cumulative number of patents (and means cumulative prior patenting knowledge) over the time period from 1 to $t, a$ is the learning-bydoing coefficient, $b$ is the sum of the R\&D expenditures coefficient, and $C E_{t}$ is the cumulative $\mathrm{R} \& \mathrm{D}$ expenditures at period $t$. The cumulative $\mathrm{R} \& \mathrm{D}$ expenditures $\left(C E_{t}\right)$ are defined as a function of past $R \& D$ investment that includes depreciation $(\delta)$ and time lag $(g)$ factors. The cumulative past $\mathrm{R} \& \mathrm{D}$ expenditures $\left(C E_{t}\right)$ can be expressed as

$$
C E_{t}=(1-\delta) \times C E_{t-1}+x_{1 t-g},
$$

where $\delta$ is the depreciation rate, and $g$ is the time lag between R\&D investment $\left(x_{1}\right)$ and its R\&D effect. For IT technology, Han (2007) assumed that a typical value that is used for the annual depreciation rate $\delta$ is $13 \%$, and there is no R\&D time lag $g$. We can take the logarithms of the both sides of Equation (1).

$$
-\ln x_{1 t}=\ln \mathrm{A}+a \times \ln \sum_{s=1}^{s=t} y_{1 s}+b \times \ln C E_{t}+\varepsilon_{t},
$$

where $\varepsilon_{t}$ denotes an error term at period $t, \hat{a}$ is the estimated value of the learning-by-doing coefficient $a$, and the cumulative effect of technological learning $\left(\widehat{x^{T L}}\right)$ is $\left(\sum_{s=1}^{s=t} y_{1 s}\right)^{\hat{a}}$.

When the technological learning curve occasionally comes to an abrupt stop, graphically, the curve jumps up, the curve jumps down, or it is truncated. This jump may cause a too large or too small learning coefficient. As technology matures, productivity growth is limited and even declines, perhaps because knowledge is forgotten or learning motivation wanes. Some scholars have argued that the depreciation of technological knowledge or experience is the main reason for negative learning (Pellegrino, Costantino, Pietroforte, \& Sancilio, 2012). Thus, when the learning coefficient is equal to or less than 0 , the learning situation is no learning or negative learning. In our model, this lack of learning or negative learning leads to very small positive effects of technological learning. In contrast, when the learning coefficient is more than 1, it represents that the learning situation is a new technology learning experience. This situation can occur if, for example, a firm can learn the upgrade or implement a new technology that can yield better productivity outcomes, which may result in a new learning curve (Ngwenyama et al., 2007). In general, the learning-by-doing coefficient $a$ is equal to or less than 1 . If the estimated value of the learning-by-doing coefficient $a$ is more than 1 , we set it to be 1 .

\subsection{Incorporating the effect of subsidy policies and technological learning into the categorical DEA model}

Calculate R\&D efficiency considering a technological learning input $\left(\widehat{x^{T L}}\right), m$ inputs $x_{i j}$ and $p$ outputs $y_{r j}$ at time $t$. To determine the total number of possible categories such that each DMU can be assigned to precisely one category, assume that there are $n \operatorname{DMU}_{j}(j=1, \ldots, n)$ that can be grouped into $L$ hierarchical categories of R\&D subsidy intensity while $L \leq n$, 
and indicate the number of DMUs in the $h^{\text {th }}$ category by $z_{h}(h=1, \ldots, L)$. It must hold that $\sum_{h=1}^{L} z_{h}=n$. The DMUs in a given category can be evaluated within the same level or a lower level of R\&D subsidy intensity. Thus, the DMUs that belong to the first category are evaluated with respect to the units in $z_{1}$, the DMUs in the second category are evaluated with respect to the units in $z_{1} \cup z_{2}$, etc. Lo and Lu (2009) suggested utilizing a slacks-based measure (SBM) to increase discrimination between efficient and inefficient DMUs. The SBM model with environmental categories of R\&D subsidy intensity $\left(z_{h}\right)$ and a technological learning input $\left(\widehat{x^{T L}}\right)$ is as follows:

$$
\text { Minimize } \rho_{0}^{z_{h}}=\frac{1-\frac{1}{m+1}\left(\sum_{i=1}^{m} \frac{s_{i}^{-}}{x_{i 0}}+\frac{s^{-T L}}{\widehat{x_{0}^{T L}}}\right)}{1+\frac{1}{p} \sum_{r=1}^{p} \frac{s_{r}^{+}}{y_{r 0}}}
$$

Subject to

$$
\begin{aligned}
& \sum_{j \in z_{h}} \lambda_{j} x_{i j}+s_{i}^{-}=x_{i 0}, \quad i=1, \ldots, m, \\
& \lambda_{j}^{T L} \widehat{x_{j}^{T L}}-s^{-T L}=\widehat{x_{0}^{T L}}, \quad j \in z_{h}, \\
& \sum_{j \in z_{h}} \lambda_{j} y_{r j}-s_{r}^{+}=y_{r 0}, \quad r=1, \ldots, p, \text { and } \\
& \lambda \geq 0, s^{-} \geq 0, s^{-T L} \geq 0, s^{+} \geq 0,
\end{aligned}
$$

where $s_{i}^{-}$is the slack in the ith input, and $s_{r}^{+}$is the slack in the $r$ th output. $\widehat{x^{T L}}$ denotes the estimated cumulative effect of technological learning, which is expressed as $\widehat{x^{T L}}=\left(\sum_{s=0}^{s=t} y_{1 s}\right)^{\hat{a}}$, and $s^{-T L}$ represents the slack in the technological learning input. By using the optimal results $\left(s_{i}^{-*}, s^{-T L^{*}}, s_{r}^{+^{*}}\right)$ in Equation (4), the efficiency score $\rho_{0}^{z_{h}^{*}}$ can be decomposed as follows:

$$
\rho_{0}^{z_{h}^{*}}=\frac{1-\sum_{i=1}^{m} \alpha_{i}-\alpha^{T L}}{1+\sum_{r=1}^{p} \beta_{r}} .
$$

Tangible input inefficiency is $\alpha_{i}=\frac{1}{m+1}\left(\frac{s_{i}^{-*}}{x_{i 0}}\right), i=1, \ldots, m$,

Intangible input inefficiency is $\alpha^{T L E}=\frac{1}{m+1}\left(\frac{s^{-T L^{*}}}{\widehat{x_{0}^{T L}}}\right)$, and

Output inefficiency is $\beta_{r}=\frac{1}{p}\left(\frac{s_{r}^{+^{*}}}{y_{r 0}}\right), r=1, \ldots, p$.

In this model, the efficiency score $\left(\rho_{0}^{z_{h}}{ }^{*}\right)$ lies between 0 and $1 ; \rho_{0}^{z_{h}{ }^{*}}=1$ represents an efficient $\mathrm{DMU}_{0}$ in the $h^{\text {th }}$ category. By using inefficiency decomposition, a manager can identify the major source of inefficiency, indicated by greater slack, and make appropriate improvements. 


\section{Defining the output, input and environmental categorical variables of $R \& D$ efficiency}

To measure R\&D efficiency, this study selected the appropriate inputs and outputs at time $t$ from R\&D activities. For the output variables, this study proposed two variables. The total number of patents $\left(y_{1}\right)$ was used to assess the value that is generated by investment in $\mathrm{R} \& \mathrm{D}$ (Guan, Zuo, Chen, \& Yam, 2016). Given that the total number of patents $\left(y_{1}\right)$ contributes revenue $\left(y_{2}\right)$ through technology or product dissemination (Hashimoto \& Haneda, 2008), the output variables included the total number of patents $\left(y_{1}\right)$ and revenue $\left(y_{2}\right)$.

The input variables were classified as intangible and tangible. Regarding intangible input, this study employed an estimate of the technological learning effect $\left(\widehat{x^{T L}}\right)$. This variable represented firms' accumulated knowledge stock or intellectual capital input. Economically tangible inputs (such as cost or expense) should be considered for R\&D efficiency assessment (Linton, Walsh, \& Morabito, 2002). For tangible input, this study considered R\&D expenditure $\left(x_{1}\right)$; selling, general and administrative (SG\&A) expenses $\left(x_{2}\right)$; and total assets $\left(x_{3}\right)$. R\&D expenditure $\left(x_{1}\right)$ can represent a firm's past R\&D efforts; thus, some scholars treat $\mathrm{R} \& \mathrm{D}$ expenditure $\left(x_{1}\right)$ as intangible. However, this study considered this knowledge in the two-factor learning experience curve. Furthermore, some studies show that learning input can effectively reduce R\&D expenditures (Grübler \& Messner, 1998). Firm R\&D investment decisions do not necessarily result in the desired outcomes (Hashimoto \& Haneda, 2008). Less R\&D expenditure input may result in greater R\&D efficiency. SG\&A expenses $\left(x_{2}\right)$ were identified as the necessary resources to develop and sustain a tacit knowledge of $\mathrm{R} \& \mathrm{D}$ activities (Lev, Radhakrishnan, \& Zhang, 2009). Because R\&D expenditures $\left(x_{1}\right)$ include research scientists' wages, and SG\&A expenses $\left(x_{2}\right)$ contain the general staff's wages (Li, 2014, 2017), this study does not consider additional labour inputs such as the number of R\&D personnel or full-time employees. In general, total assets $\left(x_{3}\right)$ represented firm size and resources that support more learning opportunities.

For the environmental categorical variable, this study chose the level of R\&D subsidy intensity $\left(z_{h}\right)$, which is adopted as an important factor of the technological learning opportunity in an environment (Kelm, Narayanan, \& Pinches, 1995). Increasing government investment in $\mathrm{R} \& \mathrm{D}$ is considered essential to ensuring knowledge-based economic growth (Hsu \& Chiang, 2001). In contrast, the level of government investment in industrial R\&D can be considered in relation to the gross domestic product (GDP) (Almus \& Czarnitzki, 2003). R\&D subsidy intensity $\left(z_{h}\right)$ is a commonly used indicator that reflects a country's industry R\&D subsidies as a percentage of its GDP. The observed country can be grouped according to the mean of the R\&D subsidy intensity $\left(z_{h}\right)$ into the two categories of low subsidy intensity $\left(z_{1}\right)$ and high subsidy intensity $\left(z_{2}\right)$.

\subsection{Data sources}

IT industry has become a major driver of economic growth (Wang, W.-R. Lin, S.-S. Lin, \& Hung, 2017). The analysis in this study was based on the estimated cumulative stock of technological learning $\left(\widehat{x^{T L}}\right)$ that results from patenting experience using a sample of 128 IT firms across 8 countries from 2008 to 2015 and on other inputs and outputs for 2015. In 
estimating the cumulative stock of technological learning $\left(\widehat{x^{T L}}\right)$, this study used R\&D expenditures $\left(x_{1}\right)$ with no missing values for the 2008 to 2015 period, and it examined the patents that were granted at the beginning of this study period and those that were granted in two or more periods. In calculating a firm's efficiency score, no missing input or output values were allowed for 2015 .

The sample data were collected from COMPUSTAT using the Global Industry Classification Standard (GICS) to search for IT companies. Our sample of firms was constructed by merging the financial data from COMPUSTAT with the patent data from the United States Patent and Trademark Office (USPTO). The US represents a huge market for product selling and technology transactions. Because patent application and protection are governed by territorial limitations, many enterprises and research institutions from all over the world submit patent applications to the US to protect their intellectual assets and research outputs. The USPTO databases have been used extensively in academic research. For the environmental categorical variable, this study uses countrywide R\&D subsidy intensity $\left(z_{h}\right)$ rather than firm-level subsidy intensity. Government incentives for $R \& D$ may take on various forms, such as $R \& D$ tax credit or financial $R \& D$ subsidies. In countries with a simple general $R \& D$ tax credit, it is likely that firm subsidy intensities are similar; where financial R\&D subsidies dominate, large differences between firms are to be expected. Financial R\&D subsidies are more effective in raising private R\&D spending and learning outcomes (Söderblom, Samuelsson, Wiklund, \& Sandberg, 2015). Government funding and other types of funding of business enterprise R\&D expenditure in the IT industry and the GDP for 2009 were released and made available by OECD sources. The observed countries are classified into two environmental categorical variables based on the R\&D subsidy intensity level (governmentfinanced $\mathrm{R} \& \mathrm{D}$ expenditures relative to GDP): low subsidy intensity $\left(z_{1}\right)$ and high subsidy intensity $\left(z_{2}\right)$. The former includes four countries: the United Kingdom, France, Norway, and Germany. The remaining four countries, Taiwan, Korea, Japan and Finland, are classified as high subsidy intensity $\left(z_{2}\right)$. To examine that the difference of two categories is significant, the nonparametric Mann-Whitney $U$ test is conducted. The resulting $Z$ statistic is shown as 2.3094 (the p-value is 0.0209 ) which reveals that the observed eight countries can be suitably grouped into two environmental categories based on the mean of the R\&D subsidy intensity $\left(z_{h}\right)$. In addition, outlier detection finds without any disturbance of extreme value on the $\mathrm{R} \& \mathrm{D}$ subsidy intensity. Note that the number of category on $\mathrm{R} \& \mathrm{D}$ subsidy intensity is limited to two for being small number of observed countries

\section{Results and discussion}

\subsection{Stage 1. Estimating the technological learning effect and data}

In the first stage, this study estimates the technological learning effect $\left(\widehat{x^{T L}}\right)$ of the analyzed 128 firms through an ordinary least-squares method before referring to the past literature to choose inputs and outputs. The descriptive statistics of the inputs, outputs and environmental categorical variables are compiled in Table 1. This table shows that the technological learning effect $\left(\widehat{x^{T L}}\right)$ was significantly different among countries. For example, the mean of the technological learning effect $\left(\widehat{x^{T L}}\right)$ in Japan was relatively high, followed by Taiwan and Norway; 
this mean in France was relatively low. These technological learning effects $\left(\widehat{x^{T L}}\right)$ were then incorporated into the categorical DEA model to evaluate R\&D efficiency. This study uses inputs and outputs to obtain additional information (e.g., patent intensity and R\&D intensity). Firm-level patent intensity and R\&D intensity are measured by dividing the total number of patents $\left(y_{1}\right)$ and $\mathrm{R} \& \mathrm{D}$ expenditures $\left(x_{1}\right)$ a firm holds by its annual revenue $\left(y_{2}\right)$. IDEX ASA located in Norway is found to have a higher patent intensity and R\&D intensity, which is affected by the lower revenue. This might be because a firm with a high patent intensity often delays introductions and thus reduces potential revenue (Moorman, Wies, Mizik, \& Spencer, 2012). In these two variables, these countries are not similarly characterized with respect to their R\&D subsidy intensity $\left(z_{h}\right)$ level. These countries can be characterized with respect to their R\&D subsidy schemes. For example, Hall and Van Reenen (2000) found that countries with high subsidy intensity levels $\left(z_{2}\right)$ have a true incremental $\mathrm{R} \& \mathrm{D}$ tax credit.

\subsection{Stage 2. Integrating the effect of subsidy policies and technological learning on $R \& D$ efficiency measures}

The categorical DEA model is utilized as follows. First, the firms in the first category (which includes only low subsidy intensity countries) are analyzed. Then, the firms in the second category (which include low subsidy intensity and high subsidy intensity countries) are aggregated and analyzed to determine the R\&D efficiency of the firms in the high subsidy intensity countries. The results of the analysis that is based on this categorization are given in Table 2. It can be observed that the firms in the United Kingdom, France and Germany have obtained the optimal efficiency score of one in the first category, which means that they are at the top performance ranking in the low subsidy intensity countries. Moreover, when the second category is applied in which all firms are included, the firms in Taiwan and Japan are the best performers, while it is more difficult for firms in the United Kingdom, France and Germany to become efficient. For the firms in low subsidy intensity countries, there is a significant difference between the means of the efficiency scores $\left(\rho^{*}\right)$ in the first and second categories.

Next, this study compared the firms' technological learning effect $\left(\widehat{x^{T L}}\right)$ with changes in their efficiency scores $\left(\rho^{*}\right)$. The results are given in Table 2 . This study used the nonparametric Wilcoxon signed-rank test to examine the null hypothesis that the overall R\&D efficiency score $\left(\rho^{*}\right)$ would show no significant difference after technological learning was considered. The resulting $Z$ statistic is 5.587839 (the $p$-value is 0.000000 ) at the one percent level of significance. Therefore, this result rejects the null hypothesis, and incorporating the technological learning effect $\left(x^{\mathrm{TL}}\right)$ into $\mathrm{R} \& \mathrm{D}$ efficiency is appropriate. Table 2 shows that the standard deviation increased, which indicates that when the technological learning effect $\left(\widehat{x^{T L}}\right)$ was considered, the differences in R\&D efficiency among countries became more apparent. This finding means that incorporating the technological learning effect $\left(\widehat{x^{T L}}\right)$ into the R\&D efficiency assessment will enhance the discriminatory power of the model. When the technological learning effect $\left(\widehat{x^{T L}}\right)$ was integrated, the average efficiency scores $\left(\rho^{*}\right)$ increased from 0.3641 to 0.6179 for firms in the low subsidy intensity countries and decreased from 0.2089 to 0.1452 for firms in the high subsidy intensity countries. 


\begin{tabular}{|c|c|c|c|c|c|c|c|c|c|c|c|c|c|c|c|c|c|}
\hline \multirow{2}{*}{ 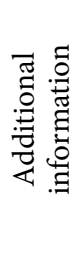 } & 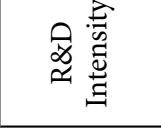 & 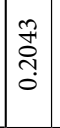 & $\mid \begin{array}{l}\hat{a} \\
0 \\
0 \\
0\end{array}$ & 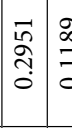 & 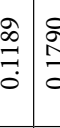 & 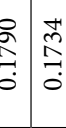 & $\mid \begin{array}{l}0 \\
0 \\
0 \\
0 \\
0 \\
0\end{array}$ & $\begin{array}{l}2 \\
\hat{8} \\
0 \\
0\end{array}$ & 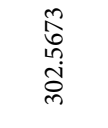 & $\mid \begin{array}{c}4 \\
\infty \\
\infty \\
-1 \\
0\end{array}$ & $\mid$\begin{tabular}{c}
$\infty$ \\
0 \\
$m$ \\
\hdashline \\
0 \\
0
\end{tabular} & 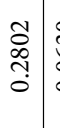 & 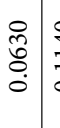 & 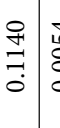 & 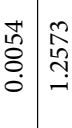 & \begin{tabular}{l}
0 \\
$\infty$ \\
\multirow{1}{*}{} \\
0 \\
0
\end{tabular} & $\begin{array}{l}\stackrel{+}{7} \\
\stackrel{0}{0}\end{array}$ \\
\hline & 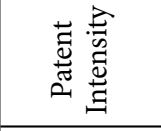 & 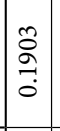 & $\overrightarrow{\hat{\theta}}$ & 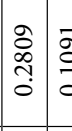 & \begin{tabular}{l|l}
$\vec{a}$ & $\overline{0}$ \\
0 & 0 \\
0 & 0 \\
0 & $n$
\end{tabular} & 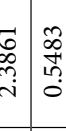 & 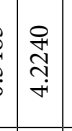 & $\begin{array}{l}\bar{\sigma} \\
\hat{\alpha} \\
i \\
i\end{array}$ & $\begin{array}{l}\stackrel{\sim}{1} \\
\infty \\
\infty \\
\infty \\
0 \\
0\end{array}$ & 寺 & $\mid \begin{array}{l}\overrightarrow{0} \\
0 \\
0 \\
0\end{array}$ & 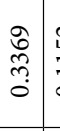 & 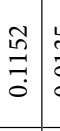 & \begin{tabular}{l|l}
$\stackrel{n}{m}$ & $\bar{c}$ \\
0 & 0 \\
0 & 0
\end{tabular} & 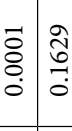 & $\begin{array}{c}\tilde{1} \\
\tilde{\delta} \\
0 \\
0\end{array}$ & $\begin{array}{l}\text { సิ } \\
\text { ○. }\end{array}$ \\
\hline \multirow{2}{*}{ 莣 } & 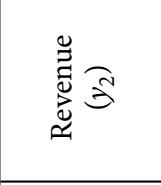 & $\mid \begin{array}{l}m \\
\tilde{n} \\
\dot{\sigma} \\
\dot{D} \\
\text { N}\end{array}$ & $\mid \begin{array}{l}8 \\
8 \\
1 \\
1 \\
0 \\
2 \\
-1\end{array}$ & 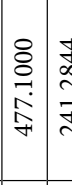 & 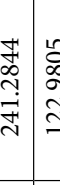 & 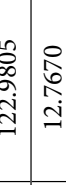 & 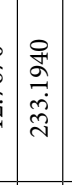 & 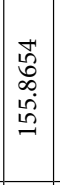 & ڤ్ & \begin{tabular}{l}
8 \\
$\infty$ \\
0 \\
$i$ \\
\\
\multirow{7}{*}{} \\
in
\end{tabular} & 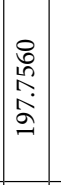 & 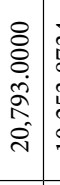 & 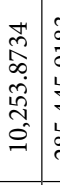 & 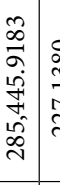 & 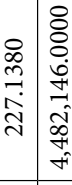 & $\begin{array}{l}\infty \\
\infty \\
0 \\
0 \\
n \\
0 \\
0 \\
n \\
0 \\
\infty \\
2 \\
\infty \\
\infty\end{array}$ & $\begin{array}{l}\text { I্+1 } \\
\text { on } \\
\infty \\
0 \\
0\end{array}$ \\
\hline & 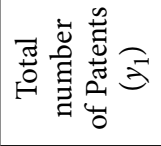 & $\mid \begin{array}{c}8 \\
\vdots \\
0 \\
0 \\
\text { in }\end{array}$ & $\begin{array}{l}8 \\
\varnothing \\
\dot{0} \\
\dot{m}\end{array}$ & 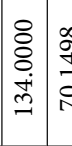 & 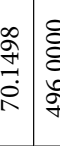 & 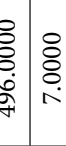 & 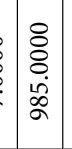 & 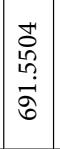 & $\begin{array}{l}8 \\
0 \\
0 \\
\infty \\
\infty \\
m\end{array}$ & $\begin{array}{l}8 \\
0 \\
1 \\
0 \\
0 \\
0 \\
0 \\
0\end{array}$ & $\mid \begin{array}{c}8 \\
\vdots \\
\vdots \\
\dot{m} \\
\end{array}$ & 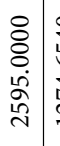 & 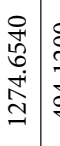 & 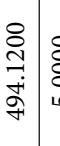 & 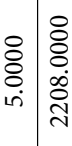 & $\begin{array}{l}0 \\
0 \\
0 \\
0 \\
\dot{0} \\
0 \\
0\end{array}$ & $\begin{array}{l}8 \\
\vdots \\
\vdots \\
\\
-1\end{array}$ \\
\hline \multirow{4}{*}{ 蒙 } & 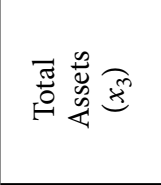 & 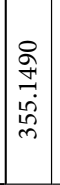 & 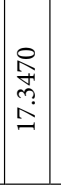 & 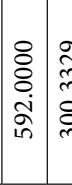 & 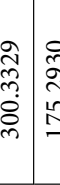 & 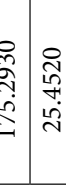 & 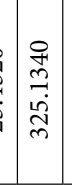 & 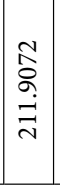 & $\begin{array}{l}\text { 울 } \\
\text { N } \\
\infty \\
\infty \\
\infty\end{array}$ & 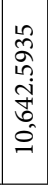 & $\mid \begin{array}{l}\dot{0} \\
\infty \\
\infty \\
\infty \\
\dot{0} \\
\infty\end{array}$ & 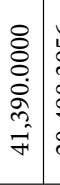 & 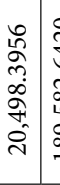 & 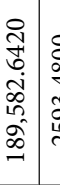 & 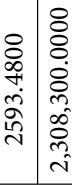 & 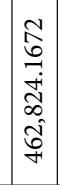 & $\begin{array}{l}\text { ते } \\
\text { h़े } \\
\text { ठें }\end{array}$ \\
\hline & 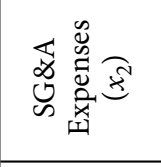 & $\begin{array}{l}\stackrel{8}{0} \\
0 \\
0 \\
\dot{0} \\
-1\end{array}$ & $\begin{array}{l}8 \\
8 \\
0 \\
0 \\
0 \\
0\end{array}$ & 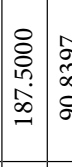 & 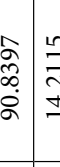 & 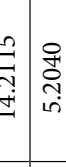 & $\begin{array}{l}\stackrel{8}{2} \\
\stackrel{\vec{n}}{\pi} \\
\tilde{i}\end{array}$ & $\mid \begin{array}{l}n \\
\infty \\
\\
\mathfrak{a} \\
\end{array}$ & 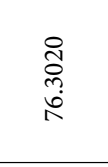 & $\mid \begin{array}{c}0 \\
0 \\
0 \\
0 \\
0 \\
0 \\
0 \\
-1\end{array}$ & 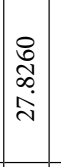 & \begin{tabular}{l}
8 \\
$\vdots$ \\
$\vdots$ \\
$g$ \\
\multirow{g}{*}{}
\end{tabular} & 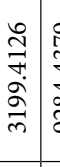 & 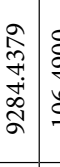 & 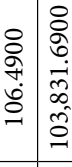 & 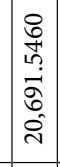 & $\begin{array}{l}8 \\
\vdots \\
\infty \\
\infty \\
\infty \\
\infty\end{array}$ \\
\hline & 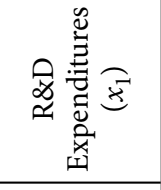 & $\mid \begin{array}{l}m \\
\hat{\sigma} \\
\vdots \\
\dot{q}\end{array}$ & $\begin{array}{l}\mathscr{D} \\
\& \\
8 \\
\dot{+}\end{array}$ & \begin{tabular}{|l|l|}
8 & 1 \\
0 & 0 \\
0 & 0 \\
0 & 0 \\
0 & 0 \\
$=$ & 0
\end{tabular} & 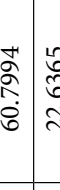 & 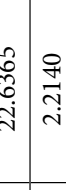 & $\begin{array}{l}0 \\
\stackrel{2}{b} \\
\dot{2} \\
\dot{y}\end{array}$ & $\mid \begin{array}{l}\infty \\
\infty \\
\infty \\
\infty \\
\infty \\
\sim\end{array}$ & 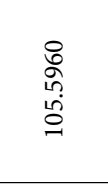 & $\begin{array}{l}\infty \\
\infty \\
\infty \\
\infty \\
i \\
i \\
\\
\end{array}$ & $\mid \begin{array}{l}0 \\
1 \\
0 \\
0 \\
0 \\
m\end{array}$ & 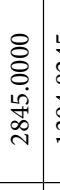 & 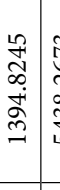 & 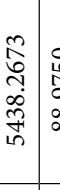 & 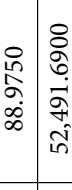 & 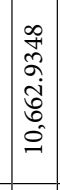 & 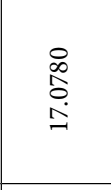 \\
\hline & 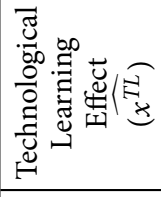 & $\begin{array}{l}m \\
\tilde{\alpha} \\
\alpha \\
\dot{m}\end{array}$ & $\mid \begin{array}{l}m \\
0 \\
\infty \\
\infty \\
0\end{array}$ & 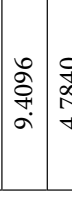 & 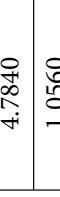 & 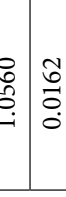 & $\begin{array}{l}\hat{2} \\
\hat{\alpha} \\
\hat{\alpha} \\
i\end{array}$ & 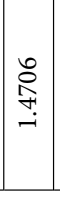 & $\begin{array}{l}8 \\
0 \\
0 \\
\infty \\
i \\
m\end{array}$ & 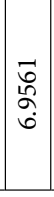 & $\begin{array}{l}\hat{L} \\
\hat{\Omega} \\
\widehat{o} \\
0\end{array}$ & $\begin{array}{c}2 \\
2 \\
\hat{n} \\
\stackrel{\lambda}{n}\end{array}$ & 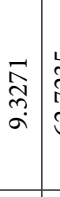 & 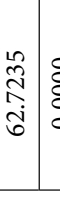 & \begin{tabular}{l|l}
0 & 8 \\
8 & 0 \\
0 & 0 \\
0 & 0 \\
0 & $=$ \\
\hdashline
\end{tabular} & 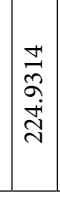 & $\begin{array}{l}0 \\
\vec{D} \\
\stackrel{0}{N}\end{array}$ \\
\hline 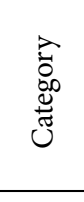 & 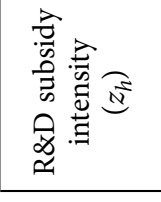 & \multicolumn{12}{|c|}{ 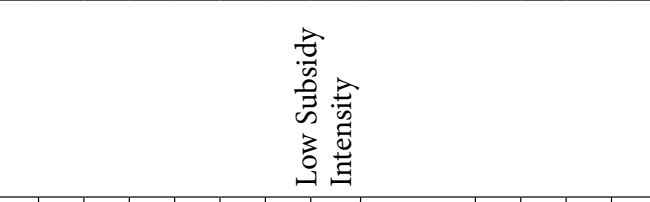 } & \multicolumn{4}{|c|}{ 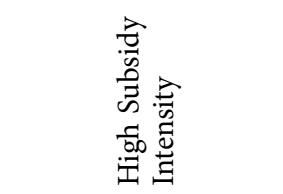 } \\
\hline \multicolumn{2}{|c|}{ 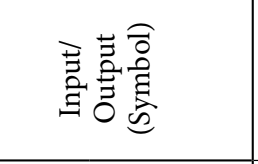 } & 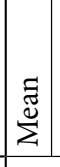 & 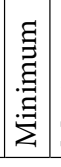 & 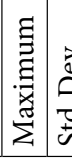 & 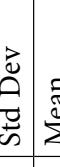 & 丞 & 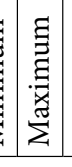 & $\begin{array}{l}\vec{D} \\
0 \\
\tilde{D} \\
\dot{\omega}\end{array}$ & 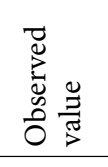 & 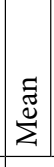 & 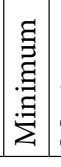 & 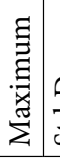 & $\begin{array}{l}\vec{D} \\
0 \\
\tilde{D} \\
\omega\end{array}$ & 胥 & 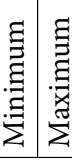 & $\begin{array}{l}\overrightarrow{\tilde{U}} \\
\stackrel{0}{\vec{n}} \\
\dot{D}\end{array}$ & 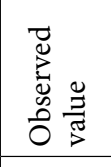 \\
\hline \multicolumn{2}{|c|}{ 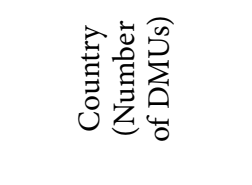 } & \multicolumn{3}{|c|}{ 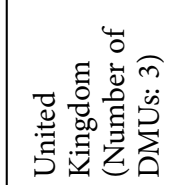 } & \multicolumn{4}{|c|}{ 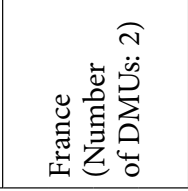 } & 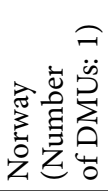 & \multicolumn{4}{|c|}{ 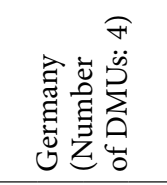 } & \multicolumn{3}{|c|}{ 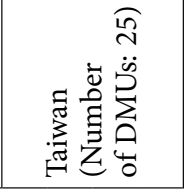 } & 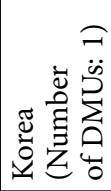 \\
\hline
\end{tabular}




\begin{tabular}{|c|c|c|c|c|c|c|c|c|c|c|c|c|c|c|c|c|c|c|c|c|c|}
\hline \multirow{2}{*}{ 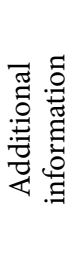 } & 量 & $\begin{array}{l}m \\
\tilde{n} \\
\tilde{o} \\
0 \\
0\end{array}$ & $\mid \begin{array}{l}\infty \\
\stackrel{0}{0} \\
\dot{0}\end{array}$ & $\begin{array}{c}\hat{\tilde{n}} \\
\hat{n} \\
\tilde{\sigma} \\
0\end{array}$ & \begin{tabular}{l}
2 \\
\multirow{2}{*}{} \\
0 \\
0
\end{tabular} & ڤ్̊ి & $\begin{array}{c}m \\
\stackrel{-}{*} \\
0\end{array}$ & $\begin{array}{c}\tilde{N} \\
\tilde{\omega} \\
\tilde{0} \\
0\end{array}$ & 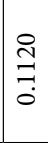 & 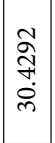 & $\begin{array}{l}\hat{a} \\
0 \\
0 \\
0\end{array}$ & 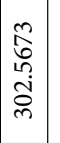 & $\begin{array}{l}\stackrel{2}{2} \\
\dot{\sigma} \\
\stackrel{2}{a}\end{array}$ & $\mid \begin{array}{l}\hat{0} \\
\hat{0} \\
0 \\
0\end{array}$ & $\begin{array}{l}\infty \\
\ddot{0} \\
\ddot{0}\end{array}$ & 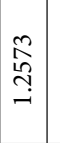 & $\begin{array}{l}\stackrel{n}{0} \\
\stackrel{2}{7} \\
0\end{array}$ & $\begin{array}{c}\stackrel{2}{Z} \\
\underset{Z}{Z} \\
i\end{array}$ & $\underset{\infty}{\infty}$ & 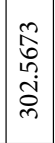 & $\begin{array}{l}\hat{0} \\
\stackrel{N}{N} \\
b \\
\dot{N}\end{array}$ \\
\hline & 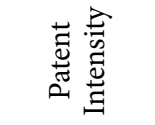 & 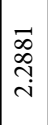 & $\begin{array}{l}\stackrel{\partial}{\sigma} \\
\dot{0} \\
\dot{0}\end{array}$ & 点 & $\begin{array}{c}\mathscr{L} \\
\stackrel{\mathbb{N}}{a} \\
\dot{m}\end{array}$ & 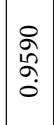 & 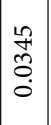 & 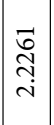 & 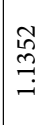 & 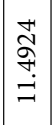 & $\vec{a}$ & $\begin{array}{l}2 \\
\infty \\
\infty \\
\infty \\
\infty \\
0 \\
-1\end{array}$ & 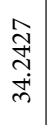 & 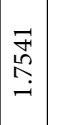 & $\begin{array}{l}\overrightarrow{0} \\
\vdots \\
0\end{array}$ & $\begin{array}{c}\mathbb{E} \\
\hat{f} \\
\stackrel{0}{0} \\
-\end{array}$ & $\begin{array}{l}\infty \\
\stackrel{1}{a} \\
a \\
i \\
i\end{array}$ & $\mid \begin{array}{c}\stackrel{2}{\vec{H}} \\
\vec{n} \\
i\end{array}$ & $\overrightarrow{8}$ & $\begin{array}{l}\stackrel{\sim}{0} \\
\infty \\
\infty \\
\infty \\
\infty \\
0\end{array}$ & $\begin{array}{l}\hat{\alpha} \\
\infty \\
\infty \\
\sigma\end{array}$ \\
\hline \multirow{2}{*}{ 䓂 } & 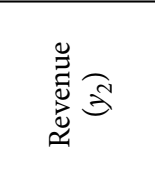 & 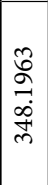 & 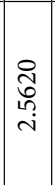 & $\begin{array}{l}8 \\
0 \\
m \\
m \\
\tilde{d} \\
0 \\
0 \\
0\end{array}$ & 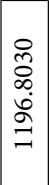 & $\begin{array}{l}n \\
\infty \\
0 \\
b \\
\dot{0} \\
\underline{\square}\end{array}$ & $\begin{array}{l}R \\
0 \\
\infty \\
\infty \\
0 \\
-1\end{array}$ & $\begin{array}{l}8 \\
0 \\
i n \\
\infty \\
0 \\
m\end{array}$ & $\begin{array}{l}\infty \\
2 \\
0 \\
0 \\
0 \\
10 \\
11\end{array}$ & 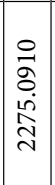 & 尽 & 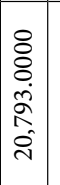 & $\begin{array}{l}n \\
\tilde{N} \\
\infty \\
\infty \\
0 \\
0 \\
0\end{array}$ & 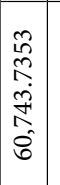 & 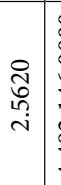 & 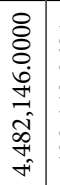 & 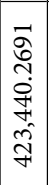 & 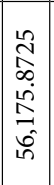 & ڤ్ & 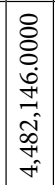 & 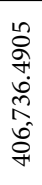 \\
\hline & 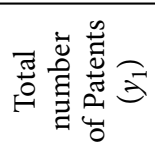 & $\begin{array}{l}\infty \\
\stackrel{\infty}{1} \\
6 \\
\infty \\
\infty \\
0 \\
0\end{array}$ & $\begin{array}{l}\stackrel{ి}{ } \\
\grave{\Xi} \\
i\end{array}$ & 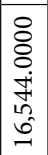 & 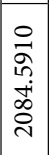 & $\begin{array}{l}8 \\
\vdots \\
\vdots \\
0 \\
\infty \\
+ \\
+\end{array}$ & $\begin{array}{l}8 \\
0 \\
0 \\
= \\
=\end{array}$ & 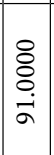 & 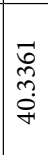 & 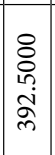 & $\begin{array}{l}8 \\
\vdots \\
\vdots \\
\dot{m}\end{array}$ & 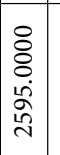 & 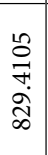 & 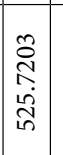 & 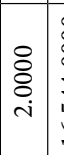 & 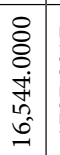 & 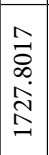 & $\mid$\begin{tabular}{c|}
$\stackrel{2}{2}$ \\
$n$ \\
$m$ \\
$i n$ \\
$i n$ \\
$i n$
\end{tabular} & 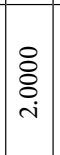 & 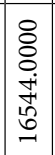 & శ్ \\
\hline \multirow{4}{*}{$\begin{array}{l}\vec{\Xi} \\
\text { 范 }\end{array}$} & 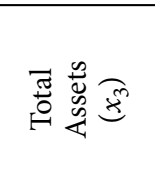 & 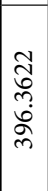 & 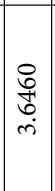 & 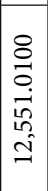 & 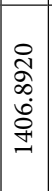 & 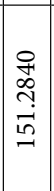 & 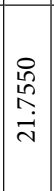 & 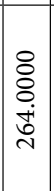 & 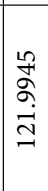 & 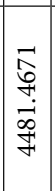 & 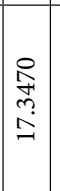 & 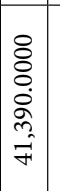 & 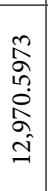 & 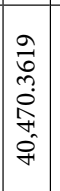 & 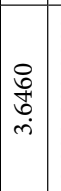 & 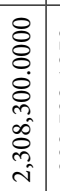 & 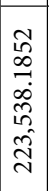 & $\mid \begin{array}{l}2 \\
2 \\
N \\
\infty \\
0 \\
0 \\
0 \\
m \\
m\end{array}$ & $\begin{array}{l}8 \\
8 \\
+ \\
0 \\
\dot{m}\end{array}$ & 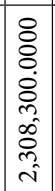 & 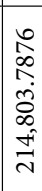 \\
\hline & 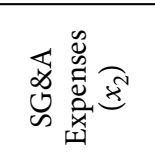 & $\frac{\tilde{2}}{2}$ & $\begin{array}{l}0 \\
\stackrel{0}{0} \\
\stackrel{0}{-1}\end{array}$ & 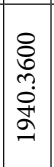 & 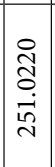 & $\begin{array}{l}R \\
0 \\
o \\
0 \\
\infty \\
0 \\
0\end{array}$ & $\begin{array}{l}0 \\
\infty \\
6 \\
\\
=\end{array}$ & $\begin{array}{l}0 \\
\infty \\
\infty \\
\hat{2} \\
\delta \\
2\end{array}$ & \begin{tabular}{l}
$F$ \\
\multirow{H}{*}{} \\
$\infty$ \\
$\infty$ \\
$\infty$ \\
$\infty$
\end{tabular} & 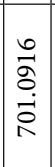 & $\begin{array}{l}\text { 오 } \\
\text { N } \\
\text { in }\end{array}$ & 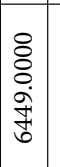 & 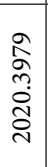 & 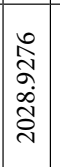 & 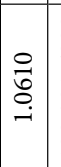 & 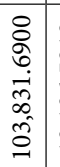 & 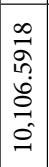 & 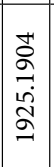 & 움 & 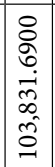 & 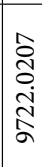 \\
\hline & 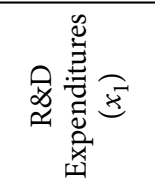 & 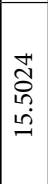 & $\begin{array}{l}\stackrel{R}{2} \\
\widehat{O} \\
\stackrel{0}{0}\end{array}$ & \begin{tabular}{|l|}
8 \\
0 \\
0 \\
$m$ \\
$m$ \\
$m$
\end{tabular} & 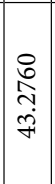 & $\begin{array}{l}\hat{0} \\
0 \\
0 \\
\tilde{n} \\
i\end{array}$ & 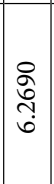 & $\begin{array}{l}8 \\
\vdots \\
\vdots \\
\dot{0} \\
m\end{array}$ & 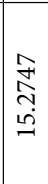 & 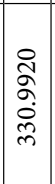 & 亲 & 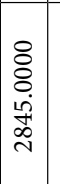 & $\begin{array}{l}0 \\
\mathscr{D} \\
\stackrel{2}{+} \\
\stackrel{\infty}{\infty} \\
\infty\end{array}$ & 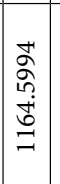 & 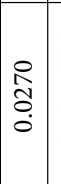 & 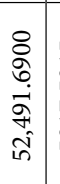 & $\begin{array}{l}n \\
0 \\
0 \\
n \\
\\
0 \\
i n\end{array}$ & $\mid \begin{array}{c}\infty \\
\tilde{N} \\
\hat{f} \\
\sigma \\
\sigma \\
0\end{array}$ & $\begin{array}{l}\stackrel{R}{\hat{I}} \\
\stackrel{0}{0}\end{array}$ & 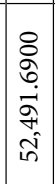 & $\begin{array}{l}\hat{N} \\
\stackrel{N}{+} \\
\stackrel{+}{=} \\
\text { in }\end{array}$ \\
\hline & 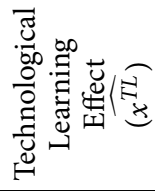 & 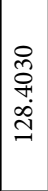 & $\vec{\Xi}$ & 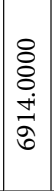 & 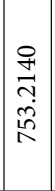 & 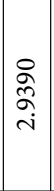 & 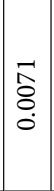 & 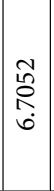 & 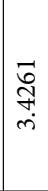 & 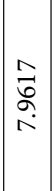 & $\mid \begin{array}{l}\tilde{0} \\
0 \\
0 \\
0 \\
0\end{array}$ & $\mid \begin{array}{c}0 \\
0 \\
0 \\
\infty \\
\infty \\
m\end{array}$ & $\begin{array}{l}\vec{I} \\
\text { तิ } \\
\text { I }\end{array}$ & 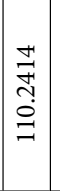 & $\mid$ & $\left|\begin{array}{c}0 \\
\delta \\
o \\
\dot{+} \\
\sigma \\
\sigma\end{array}\right|$ & 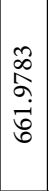 & 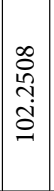 & 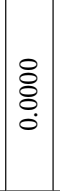 & 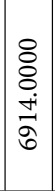 & \\
\hline 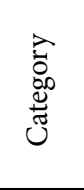 & 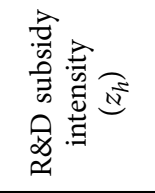 & \multicolumn{8}{|c|}{ 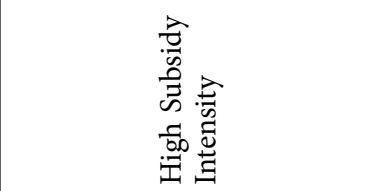 } & & & & & & & & & & & & \\
\hline \multicolumn{2}{|c|}{ 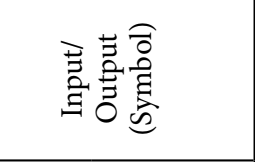 } & 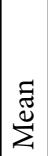 & 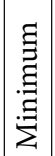 & 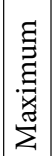 & $\begin{array}{l}\overrightarrow{\tilde{D}} \\
0 \\
\vec{D} \\
\dot{n}\end{array}$ & 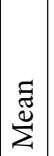 & 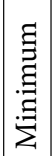 & 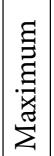 & $\begin{array}{l}\vec{D} \\
0 \\
\overrightarrow{0}\end{array}$ & 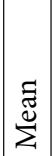 & 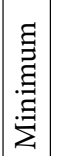 & 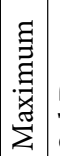 & $\begin{array}{l}\overrightarrow{\tilde{U}} \\
\dot{\vec{n}} \\
\dot{n}\end{array}$ & $\underset{\Xi}{\tilde{\Xi}}$ & 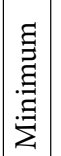 & 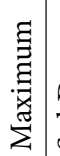 & $\begin{array}{l}\overrightarrow{\tilde{D}} \\
0 \\
\vec{D} \\
\dot{n}\end{array}$ & $\sum_{\bar{\Xi}}^{\Xi}$ & 志 & 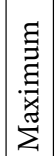 & $\begin{array}{l}\vec{U} \\
\ddot{\theta} \\
\vec{\omega}\end{array}$ \\
\hline \multicolumn{2}{|c|}{ 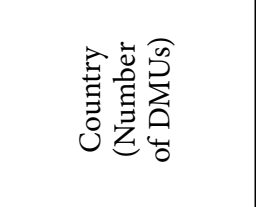 } & \multicolumn{4}{|c|}{ 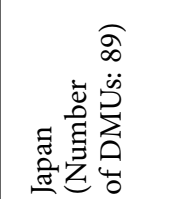 } & \multicolumn{4}{|c|}{ 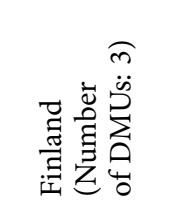 } & \multicolumn{4}{|c|}{ 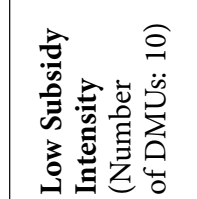 } & \multicolumn{4}{|c|}{ 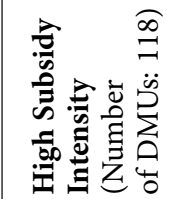 } & \multicolumn{4}{|c|}{ 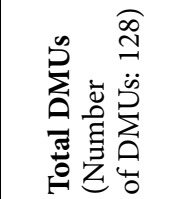 } \\
\hline
\end{tabular}


Table 2. Efficiency scores with and without integration of the technological learning effect using the categorical DEA model

\begin{tabular}{|c|c|c|c|c|c|c|}
\hline \multirow{2}{*}{$\begin{array}{c}\text { Country } \\
\text { (Number of DMUs) }\end{array}$} & \multirow[t]{2}{*}{ Statistics } & \multirow{2}{*}{$\begin{array}{l}\text { Category } \\
\text { R\&D subsidy } \\
\text { intensity } \\
\left(z_{h}\right)\end{array}$} & \multicolumn{2}{|c|}{$\begin{array}{c}\text { Efficiency score, } \\
\text { technological learning } \\
\text { effect integrated }\end{array}$} & \multicolumn{2}{|c|}{$\begin{array}{l}\text { Efficiency score, } \\
\text { technological learning } \\
\text { effect not integrated }\end{array}$} \\
\hline & & & $\begin{array}{c}\text { First } \\
\text { category }\end{array}$ & $\begin{array}{l}\text { Second } \\
\text { category }\end{array}$ & $\begin{array}{c}\text { First } \\
\text { category }\end{array}$ & $\begin{array}{l}\text { Second } \\
\text { category }\end{array}$ \\
\hline \multirow{4}{*}{$\begin{array}{l}\text { United Kingdom } \\
\text { (Number of DMUs: } 3 \text { ) }\end{array}$} & Mean & \multirow{13}{*}{$\begin{array}{l}\text { Low Subsidy } \\
\text { Intensity }\end{array}$} & 0.6206 & 0.0033 & 0.3989 & 0.0152 \\
\hline & Minimum & & 0.2174 & 0.0008 & 0.0786 & 0.0072 \\
\hline & Maximum & & 1.0000 & 0.0064 & 1.0000 & 0.0224 \\
\hline & Std Dev & & 0.3918 & 0.0029 & 0.5209 & 0.0076 \\
\hline \multirow{4}{*}{$\begin{array}{l}\text { France } \\
\text { (Number of DMUs: 2) }\end{array}$} & Mean & & 1.0000 & 0.0074 & 0.5832 & 0.2174 \\
\hline & Minimum & & 1.0000 & 0.0000 & 0.1664 & 0.0314 \\
\hline & Maximum & & 1.0000 & 0.0148 & 1.0000 & 0.4035 \\
\hline & Std Dev & & 0.0000 & 0.0105 & 0.5895 & 0.2632 \\
\hline $\begin{array}{l}\text { Norway } \\
\text { (Number of DMU: 1) }\end{array}$ & $\begin{array}{l}\text { Observed } \\
\text { value }\end{array}$ & & 1.0000 & 0.0003 & 0.0011 & 0.0002 \\
\hline \multirow{4}{*}{$\begin{array}{l}\text { Germany } \\
\text { (Number of DMUs: } 4 \text { ) }\end{array}$} & Mean & & 0.3292 & 0.0010 & 0.3192 & 0.0175 \\
\hline & Minimum & & 0.0341 & 0.0001 & 0.0491 & 0.0106 \\
\hline & Maximum & & 1.0000 & 0.0024 & 1.0000 & 0.0337 \\
\hline & Std Dev & & 0.4533 & 0.0009 & 0.4562 & 0.0109 \\
\hline \multirow{4}{*}{$\begin{array}{l}\text { Taiwan } \\
\text { (Number of DMUs: 25) }\end{array}$} & Mean & \multirow{13}{*}{$\begin{array}{l}\text { High Subsidy } \\
\text { Intensity }\end{array}$} & & 0.0804 & & 0.0820 \\
\hline & Minimum & & & 0.0000 & & 0.0001 \\
\hline & Maximum & & & 1.0000 & & 1.0000 \\
\hline & Std Dev & & & 0.2768 & & 0.2763 \\
\hline $\begin{array}{l}\text { Korea } \\
\text { (Number of DMU: 1) }\end{array}$ & \begin{tabular}{|l|}
$\begin{array}{l}\text { Observed } \\
\text { value }\end{array}$ \\
\end{tabular} & & & 0.0064 & & 0.0100 \\
\hline \multirow{4}{*}{$\begin{array}{l}\text { Japan } \\
\text { (Number of DMUs: 89) }\end{array}$} & Mean & & & 0.1695 & & 0.2515 \\
\hline & Minimum & & & 0.0000 & & 0.0008 \\
\hline & Maximum & & & 1.0000 & & 1.0000 \\
\hline & Std Dev & & & 0.2963 & & 0.2668 \\
\hline \multirow{4}{*}{$\begin{array}{l}\text { Finland } \\
\text { (Number of DMUs: } 3 \text { ) }\end{array}$} & Mean & & & 0.0133 & & 0.0670 \\
\hline & Minimum & & & 0.0000 & & 0.0056 \\
\hline & Maximum & & & 0.0386 & & 0.1460 \\
\hline & Std Dev & & & 0.0219 & & 0.0718 \\
\hline \multirow{4}{*}{$\begin{array}{l}\text { Low Subsidy Intensity } \\
\text { (Number of DMUs: 10) }\end{array}$} & Mean & & 0.6179 & & 0.3641 & \\
\hline & Minimum & & 0.0341 & & 0.0011 & \\
\hline & Maximum & & 1.0000 & & 1.0000 & \\
\hline & Std Dev & & 0.4340 & & 0.4415 & \\
\hline \multirow{4}{*}{$\begin{array}{l}\text { High Subsidy Intensity } \\
\text { (Number of DMUs: } \\
118)\end{array}$} & Mean & & & 0.1452 & & 0.2089 \\
\hline & Minimum & & & 0.0000 & & 0.0001 \\
\hline & Maximum & & & 1.0000 & & 1.0000 \\
\hline & Std Dev & & & 0.2893 & & 0.2738 \\
\hline \multirow{4}{*}{$\begin{array}{l}\text { Total DMUs } \\
\text { (Number of DMUs: } \\
128)\end{array}$} & Mean & & \multicolumn{2}{|c|}{0.1822} & \multicolumn{2}{|c|}{0.2210} \\
\hline & Minimum & & \multicolumn{2}{|c|}{0.0000} & \multicolumn{2}{|c|}{0.0001} \\
\hline & Maximum & & \multicolumn{2}{|c|}{1.0000} & \multicolumn{2}{|c|}{1.0000} \\
\hline & Std Dev & & \multicolumn{2}{|c|}{0.3266} & \multicolumn{2}{|c|}{0.2909} \\
\hline
\end{tabular}


Therefore, if the technological learning effect $\left(\widehat{x^{T L}}\right)$ is ignored, then the efficiency score $\left(\rho^{*}\right)$ of firms may be underestimated in the low subsidy intensity countries, and the efficiency score $\left(\rho^{*}\right)$ of firms may be overestimated in the high subsidy intensity countries. This result suggests that the effect of technological learning on R\&D efficiency is highly sensitive. Wilcoxon signed-rank was also used to test the null hypothesis that the overall R\&D efficiency score $\left(\rho^{*}\right)$ would show no significant difference after environmental categories of R\&D subsidy intensity $\left(z_{h}\right)$ were considered. The resulting $Z$ statistic is 3.109912 (the $p$-value is 0.001872 ) at the one percent level of significance. Therefore, this statistical result rejects the null hypothesis, and incorporating the two environmental categories of R\&D subsidy intensity $\left(z_{h}\right)$ into R\&D efficiency is appropriate. Choi and Yeniyurt (2015) believe that the high intensity of government R\&D subsidies may represent greater learning opportunities, technological learning capabilities and technological strength of the industry in a country. Lall (2000) also avers that subsidy policies play an important role in enhancing firms' technological learning because more advanced technologies tend to call for greater government support to help these firms overcome technological learning costs. Significant technological learning costs are involved in accumulating knowledge stocks and in developing R\&D output (patent counts) in most firms in the IT industry (Hanna, Boyson, \& Gunaratne, 1999). The level of subsidy intensity may play a key stimulatory role in firms' technological learning, which increases the R\&D efficiency of firms' activities (Hudon \& Traca, 2011). Governments should therefore consider increasing their budgetary appropriations or outlays for R\&D to generate greater $\mathrm{R} \& \mathrm{D}$ efficiency. However, if government subsidies are not used efficiently, the government resources that are devoted to stimulating private $R \& D$ activities are wasted. Governments that administer government-sponsored R\&D projects should therefore establish control mechanisms for tracing and managing the execution of projects at recipient firms to ensure the efficient use of subsidies (Hsu \& Hsueh, 2009).

Table 3 shows the firms with an efficiency score $\left(\rho^{*}\right)$ of 1.0 , which are considered the most R\&D-efficient firms; these firms are on the best practices frontier and can serve as benchmarks for inefficient firms. These efficient firms in the low subsidy intensity countries were relatively $R \& D$ efficient and could be referred to as better performers, not the best performers in all cases. Oxford Instruments Plc, Riber, Soitec, Idex Asa and Adva Ag Optical Networking were selected as the most appropriate benchmarks because these firms were evaluated only within the category of low subsidy intensity countries. The efficient firms in the high subsidy intensity countries are concentrated in Japan and Taiwan. The Taiwanese government devotes considerable attention to R\&D in related IT fields, demonstrated by the fact that most R\&D budgets and subsidies have gone to firms involved in the IT industry (Hsu \& Hsueh, 2009). Many Taiwanese firms in the IT industry were able to enter higher value-added R\&D activities by tapping government support (Hanna et al., 1999). Meanwhile, the government established three major science-based industrial parks with tax subsidies to support the high-tech industry's development (Hsu \& Chiang, 2001). Because of pressure from globalization trends, the demand for highly skilled workers in Taiwan's advanced technology fields has increased. Consequently, many Taiwanese workers have been forced to continue their education to upgrade their knowledge and skills or to obtain new knowledge and skills to maintain their employability and increase their long-term salary prospects (Ho \& Kuo, 2010). This pressure may enhance the R\&D efficiency of firms in Taiwan's IT industry. 
Japanese corporate leaders have long argued that R\&D expenditures are essential to maintain and increase their international competitiveness in the global IT market (El-Mashaleh, AlSmadi, Hyari, \& Rababeh, 2010). R\&D expenditures are important to obtain the new knowledge and skills that are relevant to the development of new IT products, which leads to the greater cumulative technological learning effect and further enhances R\&D efficiency. These firms were evaluated as relatively efficient in all cases and could be considered benchmarks for the best performers. Japan Aviation Electronics and Daiwabo Holdings Co Ltd, with set frequencies of 112 and 56, respectively, are benchmarks for the best performers and should be considered with special care.

Table 3. The most R\&D efficient firms in the sample that are used as benchmarks to measure the efficiency of the remaining firms

\begin{tabular}{|c|c|c|c|}
\hline Country & $\begin{array}{l}\text { Category } \\
\text { R\&D subsidy } \\
\text { intensity }\left(z_{h}\right)\end{array}$ & Company Name & $\begin{array}{l}\text { Reference set } \\
\text { frequency }\end{array}$ \\
\hline $\begin{array}{l}\text { United Kingdom } \\
\text { (No. of efficient DMUs: 1) }\end{array}$ & \multirow{5}{*}{$\begin{array}{l}\text { Low Subsidy } \\
\text { Intensity }\end{array}$} & Oxford Instruments Plc & 3 \\
\hline \multirow{2}{*}{$\begin{array}{l}\text { France } \\
\text { (No. of efficient DMUs: 2) }\end{array}$} & & Riber & 6 \\
\hline & & Soitec & 6 \\
\hline $\begin{array}{l}\text { Norway } \\
\text { (No. of efficient DMUs: } 1 \text { ) }\end{array}$ & & Idex Asa & 1 \\
\hline $\begin{array}{l}\text { Germany } \\
\text { (No. of efficient DMUs: } 1 \text { ) }\end{array}$ & & Adva Ag Optical Networking & 4 \\
\hline \multirow{2}{*}{$\begin{array}{l}\text { Taiwan } \\
\text { (No. of efficient DMUs: } 2 \text { ) }\end{array}$} & \multirow{10}{*}{$\begin{array}{l}\text { High Subsidy } \\
\text { Intensity }\end{array}$} & Quanta Computer Inc & 18 \\
\hline & & Hon Hai Precision Ind Co Ltd & 1 \\
\hline \multirow{8}{*}{$\begin{array}{l}\text { Japan } \\
\text { (No. of efficient DMUs: 8) }\end{array}$} & & Japan Aviation Electronics & 112 \\
\hline & & Daiwabo Holdings Co Ltd & 56 \\
\hline & & Mitsui High-Tec Inc & 9 \\
\hline & & Planet Inc & 4 \\
\hline & & Jastec Co Ltd & 2 \\
\hline & & Shinko Electric Industries & 2 \\
\hline & & Nihon Dempa Kogyo Co Ltd & 1 \\
\hline & & Access Co Ltd & 1 \\
\hline
\end{tabular}

The adjustments of the inputs and outputs at the country level can be identified from the difference in rates, as seen in Table 4 . In the low subsidy intensity countries, most firms show the greatest difference in the number of patents. Most firms in the high subsidy intensity countries reflect the greatest difference in the rates between the technological learning effect $\left(\widehat{x^{T L}}\right)$ and the number of patents. Table 4 also identifies the important sources of inefficiency using decomposition. This study finds that the important sources of R\&D inefficiency in the IT industry are a shortfall in the number of patents and a lack of technological learning input. Government policy makers must introduce subsidies to stimulate the technological learning of local firms, which increases the number of patents in the IT industry. 


\begin{tabular}{|c|c|c|c|c|c|c|c|c|c|c|c|c|c|c|c|c|c|c|c|}
\hline 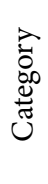 & 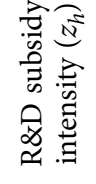 & \multicolumn{4}{|c|}{ 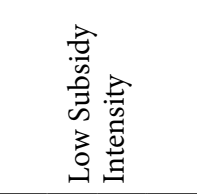 } & \multicolumn{7}{|c|}{ 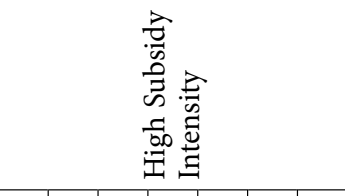 } & & & & & & & \\
\hline \multirow{2}{*}{ 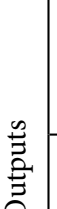 } & 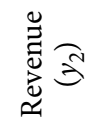 & 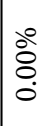 & 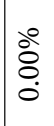 & 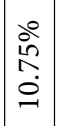 & $\begin{array}{l}\stackrel{0}{\infty} \\
\infty \\
i \\
i n\end{array}$ & 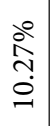 & 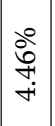 & 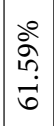 & $\begin{array}{l}\dot{0} \\
0 \\
\infty \\
\dot{m} \\
\dot{m}\end{array}$ & 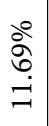 & $\begin{array}{l}\stackrel{0}{ } \\
i n \\
\infty \\
i n \\
\text { in }\end{array}$ & ঐ̊ & $\begin{array}{l}0 \\
\stackrel{0}{0} \\
\infty \\
\infty\end{array}$ & $\begin{array}{l}\text { 今े } \\
\text { in } \\
\text { bे }\end{array}$ & $\begin{array}{l}\stackrel{0}{0} \\
\stackrel{n}{n}\end{array}$ & $\begin{array}{l}\stackrel{0}{\circ} \\
\infty \\
\stackrel{0}{0} \\
= \\
=\end{array}$ & $\begin{array}{l}\stackrel{0}{\dagger} \\
\stackrel{0}{+} \\
\text { in }\end{array}$ & $\begin{array}{l}\stackrel{8}{\circ} \\
\stackrel{1}{+} \\
= \\
=\end{array}$ & 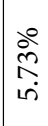 \\
\hline & $\vec{D}$ & & & & 0 & $\circ$ & 0 & 0 & 0 & $\circ$ & $\circ$ & & & & & ๑ & & $\therefore$ & \\
\hline
\end{tabular}

存

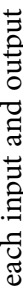

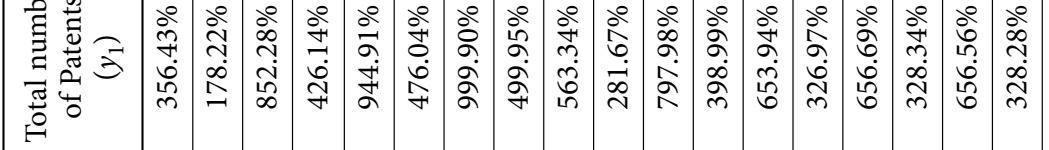

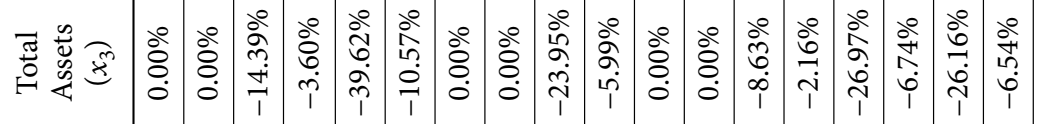

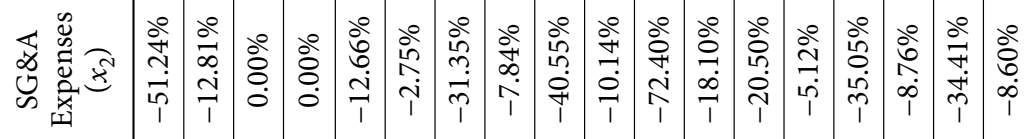

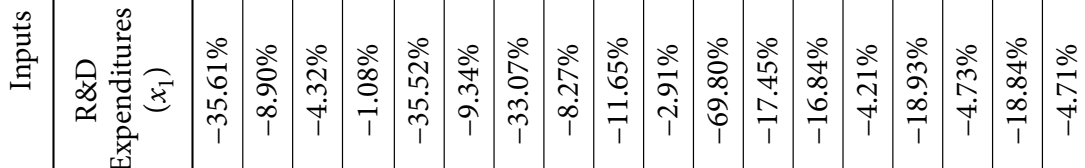

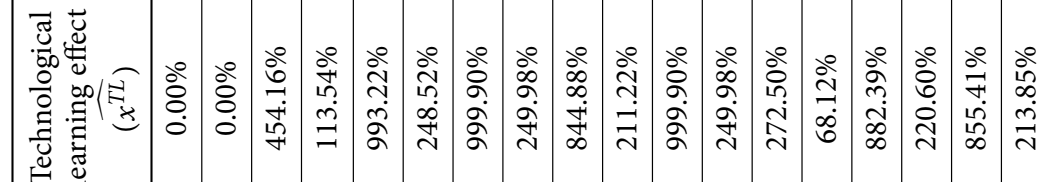
r.

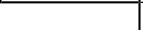

\begin{tabular}{|c|c|c|c|c|c|c|c|c|c|c|c|c|c|c|c|c|c|}
\hline 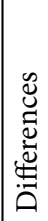 & 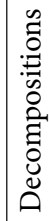 & 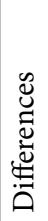 & 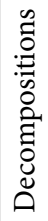 & 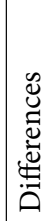 & 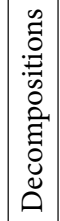 & 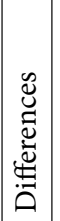 & 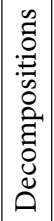 & 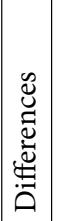 & 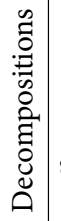 & 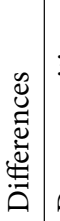 & 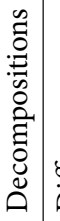 & 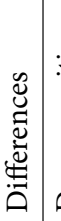 & 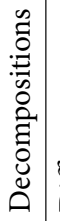 & 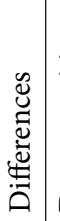 & 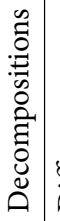 & 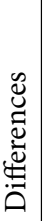 & 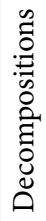 \\
\hline
\end{tabular}

\begin{tabular}{|c|c|c|c|c|c|c|c|c|c|}
\hline 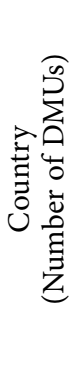 & 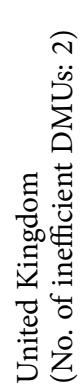 & 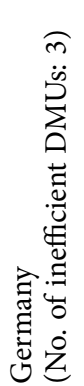 & 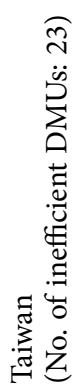 & 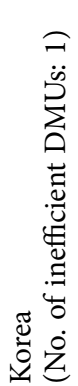 & 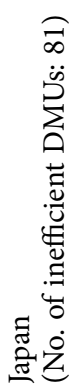 & 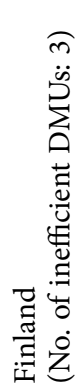 & 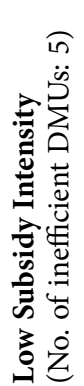 & 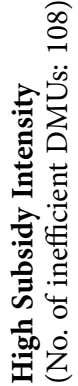 & \\
\hline
\end{tabular}




\section{Conclusions}

Government subsidies can help firms develop technological learning, and this technological learning input has a significant impact on firms' R\&D efficiency. Such a relationship is difficult to investigate using traditional approaches. Thus, a two-stage approach was developed in this paper that incorporates the effects of subsidy policies and technological learning into the DEA structure to assess R\&D efficiency. This approach demonstrates to policymakers and managers that government R\&D subsidy intensity can facilitate firms' technological learning input, which also enhances firms' R\&D efficiency. The empirical results can also help inefficient firms identify an appropriate benchmark under the same subsidy intensity and use appropriate strategies to improve their R\&D efficiency. The benchmark firms in the low subsidy intensity and high subsidy intensity countries, respectively, are concentrated in France and Japan. This study decomposed the efficiency scores by identifying the inefficiency of the most important variable in the shortage, which is caused by the number of patents and technological learning, using a slack variable analysis of inefficient firms to improve the input and output of their R\&D activities. These inefficient firms need better knowledge management to increase the technological learning input and the number of patents. R\&D subsidies are a very important industrial policy tool in many countries; they incentivize and channel R\&D investments into technological learning, which increases the number of patents in the IT industry. Government R\&D subsidies can help give firms more technology learning opportunities. Through continuous learning, firms have the ability to effectively initiate the creation and accumulation of technological capabilities. Thus, governments should consider providing more funding for $\mathrm{R} \& \mathrm{D}$ activities to achieve greater $\mathrm{R} \& \mathrm{D}$ efficiency. However, if government $\mathrm{R} \& \mathrm{D}$ subsidies are not used efficiently, the government resources that are devoted to stimulating private R\&D activities are wasted. Governments that implement and administer government-sponsored $\mathrm{R} \& \mathrm{D}$ projects should also establish appropriate mechanisms for tracking and managing the project execution in recipient firms so that their subsidies are used efficiently. Corporate decision makers must be able to effectively manage the money that is invested in technological learning to create a continuous learning cycle and to seek an adequate $\mathrm{R} \& \mathrm{D}$ subsidy environment.

Considering the vast number of data presented, which were constructed by combining data from the COMPUSTAT, USPTO and OECD databases, this study cannot include more detailed data (e.g., IT subsectors). We recommend that future research examine whether different subsectors of IT affect R\&D and patent intensities and further explore how this result is robust to different patenting propensities in various IT subsectors. However, future research on these topics should compare multiple databases. In addition, future researchers should consider the use of the dynamic DEA structure to measure changes in R\&D efficiency over a long time period and to investigate the effects of important factors on technological learning or R\&D efficiency in different countries.

\section{Acknowledgements}

L.-T. Yeh wishes to thank the Ministry of Science and Technology, Taiwan, which provided funding through contract MOST 103-2410-H-033 -043. 


\section{Funding}

This work was supported by the Ministry of Science and Technology, Taiwan [grant numbers MOST 103-2410-H-033-043].

\section{Author contributions}

LTY and DSC were responsible for designing the research. LTY was responsible for collecting and analyzing the data, interpreting the result, and writing the article.

\section{Disclosure statement}

The authors declare that they have no competing financial, professional, or personal interests from other parties that relate to the research described in this paper.

\section{References}

Almus, M., \& Czarnitzki, D. (2003). The effects of public R\&D subsidies on firms' innovation activities. Journal of Business \& Economic Statistics, 21(2), 226-236. https://doi.org/10.1198/073500103288618918

Barreto, L., \& Kypreos, S. (2004). Endogenizing R\&D and market experience in the "bottom-up" energysystems ERIS model. Technovation, 24(8), 615-629. https://doi.org/10.1016/S0166-4972(02)00124-4

Chen, Y.-H., Wen, X.-W., Wang, B., \& Nie, P.-Y. (2017). Agricultural pollution and regulation: How to subsidize agriculture? Journal of Cleaner Production, 164, 258-264. https://doi.org/10.1016/j.jclepro.2017.06.216

Choi, J., \& Yeniyurt, S. (2015). Contingency distance factors and international research and development (R\&D), marketing, and manufacturing alliance formations. International Business Review, 24(6), 1061-1071. https://doi.org/10.1016/j.ibusrev.2015.04.007

Chung, S. (2001). The learning curve and the yield factor: the case of Korea's semiconductor industry. Applied Economics, 33(4), 473-483. https://doi.org/10.1080/00036840122474

Coccia, M. (2009). What is the optimal rate of R\&D investment to maximize productivity growth? Technological Forecasting and Social Change, 76(3), 433-446. https://doi.org/10.1016/j.techfore.2008.02.008

Czarnitzki, D., \& Hussinger, K. (2018). Input and output additionality of R\&D subsidies. Applied Economics, 50(12), 1324-1341. https://doi.org/10.1080/00036846.2017.1361010

Dodgson, M. (1991). The management of technological learning: lessons from a biotechnology company. Berlin: Walter de Gruyter. https://doi.org/10.1515/9783110867749

El-Mashaleh, M., Al-Smadi, B. M., Hyari, K. H., \& Rababeh, S. M. (2010). Safety management in the Jordanian construction industry. Jordan Journal of Civil Engineering, 4(1), 117-120.

Grübler, A., \& Messner, S. (1998). Technological change and the timing of mitigation measures. Energy Economics, 20(5), 495-512. https://doi.org/10.1016/S0140-9883(98)00010-3

Guan, J., Zuo, K., Chen, K., \& Yam, R. C. M. (2016). Does country-level R\&D efficiency benefit from the collaboration network structure? Research Policy, 45(4), 770-784.

https://doi.org/10.1016/j.respol.2016.01.003

Haas, D. A., \& Murphy, F. H. (2003). Compensating for non-homogeneity in decision-making units in data envelopment analysis. European Journal of Operational Research, 144(3), 530-544.

https://doi.org/10.1016/S0377-2217(02)00139-X 
Hall, B., \& Van Reenen, J. (2000). How effective are fiscal incentives for R\&D? A review of the evidence. Research Policy, 29(4), 449-469. https://doi.org/10.1016/S0048-7333(99)00085-2

Han, Y. J. (2007). Measuring industrial knowledge stocks with patents and papers. Journal of Informetrics, 1(4), 269-276. https://doi.org/10.1016/j.joi.2007.06.001

Hanna, N., Boyson, S., \& Gunaratne, S. (1999). The east Asian miracle and information technology: strategic management of technological learning. Washington: World Bank Publications.

Hashimoto, A., \& Haneda, S. (2008). Measuring the change in R\&D efficiency of the Japanese pharmaceutical industry. Research Policy, 37(10), 1829-1836. https://doi.org/10.1016/j.respol.2008.08.004

Hitt, M. A., Ireland, R. D., \& Lee, H. U. (2000). Technological learning, knowledge management, firm growth and performance: an introductory essay. Journal of Engineering and Technology Management, 17(3), 231-246. https://doi.org/10.1016/S0923-4748(00)00024-2

Ho, L. A., \& Kuo, T. H. (2010). How can one amplify the effect of e-learning? An examination of hightech employees' computer attitude and flow experience. Computers in Human Behavior, 26(1), 2331. https://doi.org/10.1016/j.chb.2009.07.007

Hsu, C. W., \& Chiang, H. C. (2001). The government strategy for the upgrading of industrial technology in Taiwan. Technovation, 21(2), 123-132. https://doi.org/10.1016/S0166-4972(00)00029-8

Hsu, F. M., \& Hsueh, C. C. (2009). Measuring relative efficiency of government-sponsored R\&D projects: a three-stage approach. Evaluation and Program Planning, 32(2), 178-186. https://doi.org/10.1016/j.evalprogplan.2008.10.005

Hudon, M., \& Traca, D. (2011). On the efficiency effects of subsidies in microfinance: an empirical inquiry. World Development, 39(6), 966-973. https://doi.org/10.1016/j.worlddev.2009.10.017

Kelm, K. M., Narayanan, V., \& Pinches, G. E. (1995). Shareholder value creation during R\&D innovation and commercialization stages. Academy of Management Journal, 38(3), 770-786. https://doi.org/10.2307/256745

Kessler, E. H., Bierly, P. E., \& Gopalakrishnan, S. (2000). Internal vs. external learning in new product development: effects on speed, costs and competitive advantage. $R \& D$ Management, 30(3), 213-224. https://doi.org/10.1111/1467-9310.00172

Kouvaritakis, N., Soria, A., \& Isoard, S. (2000). Modelling energy technology dynamics: methodology for adaptive expectations models with learning by doing and learning by searching. International Journal of Global Energy Issues, 14(1-4), 104-115. https://doi.org/10.1504/IJGEI.2000.004384

Lall, S. (2000). The technological structure and performance of developing country manufactured exports, 1985-98. Oxford Development Studies, 28(3), 337-369. https://doi.org/10.1080/713688318

Lev, B., Radhakrishnan, S., \& Zhang, W. (2009). Organization capital. Abacus, 45(3), 275-298. https://doi.org/10.1111/j.1467-6281.2009.00289.x

Li, N. (2014). Labor market peer firms. University of Chicago, The University of Chicago Booth School of Business.

Li, N. (2017, November 1). Who are my peers? Labor market peer firms through employees' internet co-search patterns (Rotman School of Management Working Paper No. 2558271). Retrieved from https://papers.ssrn.com/sol3/papers.cfm?abstract_id=2558271

Linton, J. D., Walsh, S. T., \& Morabito, J. (2002). Analysis, ranking and selection of R\&D projects in a portfolio. R\&D Management, 32(2), 139-148. https://doi.org/10.1111/1467-9310.00246

Lo, S.-F., \& Lu, W.-M. (2009). An integrated performance evaluation of financial holding companies in Taiwan. European Journal of Operational Research, 198(1), 341-350.

https://doi.org/10.1016/j.ejor.2008.09.006 
Lyu, K., Bian, Y., \& Yu, A. (2018). Environmental efficiency evaluation of industrial sector in China by incorporating learning effects. Journal of Cleaner Production, 172, 2464-2474.

https://doi.org/10.1016/j.jclepro.2017.11.163

Moorman, C., Wies, S., Mizik, N., \& Spencer, F. J. (2012). Firm innovation and the ratchet effect among consumer packaged goods firms. Marketing Science, 31(6), 934-951. https://doi.org/10.1287/mksc.1120.0737

Nakata, T., Sato, T., Wang, H., Kusunoki, T., \& Furubayashi, T. (2011). Modeling technological learning and its application for clean coal technologies in Japan. Applied Energy, 88(1), 330-336. https://doi.org/10.1016/j.apenergy.2010.05.022

Ngwenyama, O., Guergachi, A., \& McLaren, T. (2007). Using the learning curve to maximize IT productivity: a decision analysis model for timing software upgrades. International Journal of Production Economics, 105(2), 524-535. https://doi.org/10.1016/j.ijpe.2006.02.013

Nie, P.-Y., Wang, C., Chen, Y.-H., \& Yang, Y.-C. (2018). Effects of switching costs on innovative investment. Technological and Economic Development of Economy, 24(3), 933-949. https://doi.org/10.3846/tede.2018.1430

Nie, P.-Y., \& Wang, C. (2019). An analysis of cost-reduction innovation under capacity constrained inputs. Applied Economics, 51(6), 564-576. https://doi.org/10.1080/00036846.2018.1497850

Okamuro, H., \& Nishimura, J. (2018). Whose business is your project? A comparative study of different subsidy policy schemes for collaborative R\&D. Technological Forecasting and Social Change, 127, 85-96. https://doi.org/10.1016/j.techfore.2017.07.017

Papineau, M. (2006). An economic perspective on experience curves and dynamic economies in renewable energy technologies. Energy Policy, 34(4), 422-432. https://doi.org/10.1016/j.enpol.2004.06.008

Patibandla, M., \& Petersen, B. (2002). Role of transnational corporations in the evolution of a high-tech industry: the case of India's software industry. World Development, 30(9), 1561-1577. https://doi.org/10.1016/S0305-750X(02)00060-8

Peters, T., \& Waterman, R. (1985). In search of excellence: Lessons from America's best-run companies. Journal of Business Ethics, 4(1), 70-80.

Pellegrino, R., Costantino, N., Pietroforte, R., \& Sancilio, S. (2012). Construction of multi-storey concrete structures in Italy: patterns of productivity and learning curves. Construction Management and Economics, 30(2), 103-115. https://doi.org/10.1080/01446193.2012.660776

Söderblom, A., Samuelsson, M., Wiklund, J., \& Sandberg, R. (2015). Inside the black box of outcome additionality: effects of early-stage government subsidies on resource accumulation and new venture performance. Research Policy, 44(8), 1501-1512. https://doi.org/10.1016/j.respol.2015.05.009

Söderholm, P., \& Sundqvist, T. (2007). Empirical challenges in the use of learning curves for assessing the economic prospects of renewable energy technologies. Renewable Energy, 32(15), 2559-2578. https://doi.org/10.1016/j.renene.2006.12.007

Sohn, S. Y., \& Moon, T. H. (2004). Decision tree based on data envelopment analysis for effective technology commercialization. Expert Systems with Applications, 26(2), 279-284. https://doi.org/10.1016/j.eswa.2003.09.011

Wang, C., Nie, P.-Y., Peng, D.-H., \& Li, Z.-H. (2017). Green insurance subsidy for promoting clean production innovation. Journal of Cleaner Production, 148, 111-117. https://doi.org/10.1016/j.jclepro.2017.01.145

Wang, E. C., \& Huang, W. (2007). Relative efficiency of R\&D activities: a cross-country study accounting for environmental factors in the DEA approach. Research Policy, 36(2), 260-273. https://doi.org/10.1016/j.respol.2006.11.004 
Wang, Y.-H., Lin, W.-R., Lin, S.-S., \& Hung, J.-C. (2017). How does patent litigation influence dynamic risk for market competitors? Technological and Economic Development of Economy, 23(5), 780-793. https://doi.org/10.3846/20294913.2015.1074949

Wang, X., Li, H., Li, R., Li, B., \& Zhu, D. (2016). Research on the cost forecast of China's photovoltaic industry. $R \& D$ Management, 46(1), 3-12. https://doi.org/10.1111/radm.12102

Wong, C.-Y., \& Govindaraju, V. C. (2012). Technology stocks and economic performance of government-linked companies: the case of Malaysia. Technological and Economic Development of Economy, 18(2), 248-261. https://doi.org/10.3846/20294913.2012.688313

Wright, T. P. (1936). Factors affecting the cost of airplanes. Journal of Aeronautical Sciences, 3(4), 122128. https://doi.org/10.2514/8.155

Yang, Y.-C., Nie, P.-Y., Liu, H.-T., \& Shen, M.-H. (2018). On the welfare effects of subsidy game for renewable energy investment: toward a dynamic equilibrium model. Renewable Energy, 121, 420428. https://doi.org/10.1016/j.renene.2017.12.097 